\title{
Standardized Step-by-step Technique Using Surgical Landmarks in Robotic Lateral Pelvic Lymph Node Dissection
}

\author{
Jung Hoon Bae, Wooree Koh, Hyun Ho Kim, Yoon Suk Lee \\ Division of Colorectal Surgery, Department of Surgery, Seoul St. Mary's Hospital, College of Medicine, The Catholic University of Korea, \\ Seoul, Korea
}

We aimed to show that a standardized step-by-step robotic approach using surgical landmarks could make lateral pelvic lymph node dissection (LPND) less complicated. We performed robot-assisted LPND consisting of 4 steps using surgical landmarks. The first step is a dissection of uretero-hypogastric fascia, which envelopes the ureter and the hypogastric nerve. The second step is a dissection of the medial side of the external iliac vein located at the lateral border of the obturator lymph nodes (LNs) group. The third step is a dissection of the vesico-hypogastric fascia, which is at the medial border of the obturator LNs group. The final step is a dissection of the internal iliac artery until the Alcock's canal. Indocyanine green was injected just before surgery around the dentate line to identify the lateral pelvic LNs. Standardization using a robotic approach for LPND guided by surgical landmarks allows a safer and more effective surgery.

Keywords: Rectal neoplasms; Lateral pelvic lymph node dissection; Robotic surgical procedures

\section{INTRODUCTION TO THE VIDEO}

The oncological benefit of lateral pelvic lymph node dissection (LPND) is a controversial issue in rectal cancer treatment. Preoperative chemoradiotherapy (CRT) followed by total mesorectal excision (TME) is the standard treatment for advanced low rectal cancer in Western countries [1]. In contrast, LPND combined with TME without CRT is a routine procedure in clinical stage II and III rectal cancer in Japan [2]. Recently, CRT followed by TME with selective LPND only for patients with enlarged lateral lymph node (LN) has been proposed as an alternative treatment option. This strategy aims to gain oncological benefit while minimizing surgical morbidity from overtreatment $[3,4]$.

Received: May 20,2020 - Revised: Jul 18, 2020 - Accepted: Aug 5, 2020 Correspondence to: Yoon Suk Lee, M.D.

Division of Colorectal Surgery, Department of Surgery, Seoul St. Mary Hospital, College of Medicine, The Catholic University of Korea, 222 Banpodaero, Seocho-gu, Seoul 06591, Korea

Tel: +82-2-2258-6095, Fax: +82-2-595-2282

E-mail:yslee@catholic.ac.kr

ORCID: https://orcid.org/0000-0002-1849-2774

(c) 2021 The Korean Society of Coloproctology

This is an open-access article distributed under the terms of the Creative Commons Attribution NonCommercial License (https://creativecommons.org/licenses/by-nc/4.0) which permits unrestricted noncommercial use, distribution, and reproduction in any medium, provided the original work is properly cited.
However, LPND is not widely accepted because of its complexity, intricacy, and complications (urinary or sexual dysfunction). Moreover, while the technical aspects of TME are well established, LPND is not yet standardized.

The robotic system has several advantages. It offers endo-wrist, 3-dimensional image, and firefly mode, which allows a surgeon to perform a more accurate dissection, thus, reducing the risk of complications [5]. Defining and following surgical landmarks is a useful way to make surgery simpler and easier. Recently, indocyanine green (ICG) has been widely used in many surgical fields. ICG may determine the lymphatic flow and perfusion status [6, 7]. Using ICG can identify the location of lateral pelvic LN.

The aim of this video is to introduce a standardized step-by-step technique using surgical landmarks in LPND by a robotic approach (Supplementary video clip 1).

\section{PATIENT}

The patient was a 49-year-old male with a body mass index of $24.9 \mathrm{~kg} / \mathrm{m}^{2}$. He was diagnosed with a well-differentiated adenocarcinoma of the rectum. The lower margin of the tumor was 4 $\mathrm{cm}$ from the anal verge. The initial magnetic resonance imaging (MRI) of the rectum showed clinical stage T4aN2 and bilaterally enlarged obturator LNs (largest short-axis diameter of $8 \mathrm{~mm}$ ) 
Volume 37, Number 1, 2021

Ann Coloproctol 2021;37(1):58-60



(Fig. 1A, B). As a preoperative CRT, radiation was administered to the whole pelvis at a dose of $33 \mathrm{~Gy}$ in 10 fractions for 2 weeks. After completion of radiotherapy, the pelvic MRI showed downstaging to T3N1; however, there was no change in the size of lateral LNs even after CRT (Fig. 2). We performed radical surgery with bilateral LPND. This video article was approved by the Institutional Review Board of the Ethics Committee of College of Medicine, The Catholic University of Korea (No. KC20ZASI0363). Written informed consent of patient was obtained for publication of this report and accompanying images and video.

\section{SURGICAL TECHNIQUE}

After induction of general anesthesia, 1-mL ICG was injected
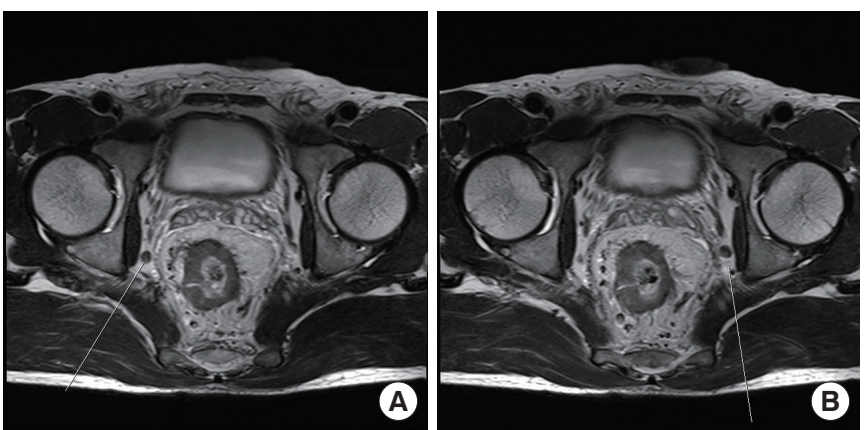

Fig. 1. Rectal magnetic resonance imaging before chemoradiation. Enlarged lymph nodes were observed in bilateral pelvic area. (A) Right. (B) Left.

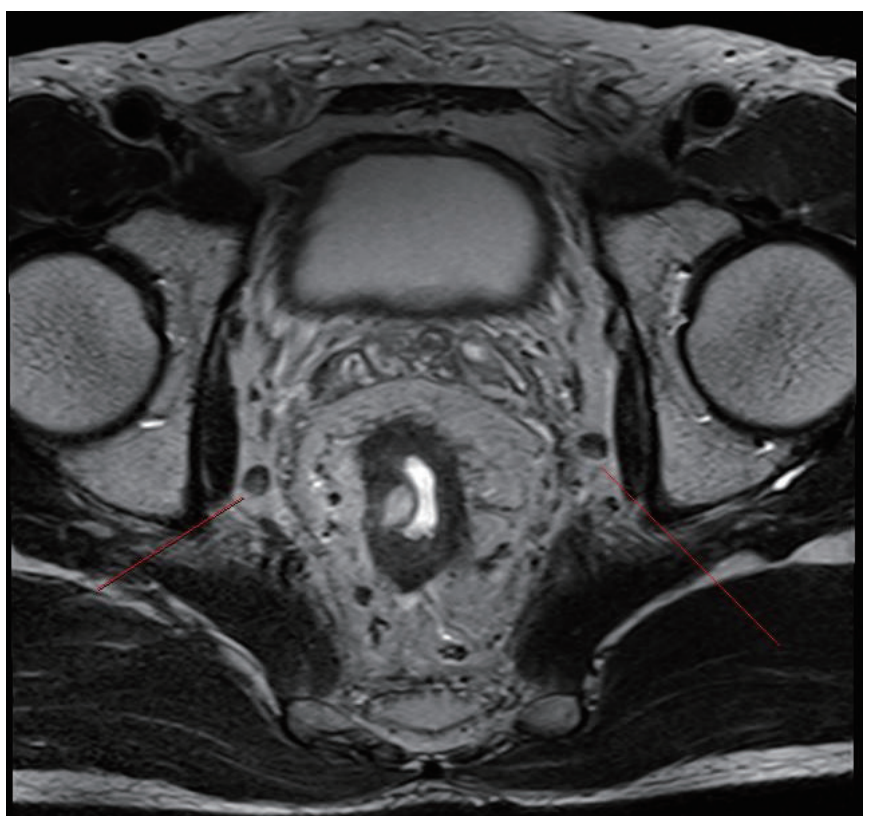

Fig. 2. Rectal magnetic resonance imaging after completion of chemoradiation. Enlarged lymph nodes were still shown in bilateral pelvic area. around the dentate line. We used the hybrid approach with laparoscopic and robotic system. Port sites are shown in Fig. 3. GelPOINT (Applied Medical, Rancho Santa Margarita, CA, USA) was inserted through a 4-cm long umbilical mini-laparotomy site. Seven ports were placed as follows: two ports in GelPOINT, an $8-\mathrm{mm}$ robotic trocar through one of port in GelPOINT, a 12-mm robotic trocar in the right-lower quadrant, two 8-mm robotic trocars in the left-upper quadrant, one assistant trocar each on the right-upper quadrant, and the left-lower quadrant. The high ligation of inferior mesenteric artery and splenic flexure mobilization was performed using laparoscopic technique. The robotic system was applied to TME and LPND. The da Vinci Xi Surgical System (Intuitive Surgical Inc., Sunnyvale, CA, USA) was used in this operation with the robotic cart docked from the left side of the $\neg$ patient. TME was completed and then LPND was started. The first robot arm (with tip-up fenestrated grasper) applied major traction to obtain an adequate surgical view. The second arm (with fenestrated bipolar forceps) was used to grasp and retract the tissue, and occasionally to control bleeding. The third arm (with monopolar curved scissor) was used for dissection (Fig. 4).

\section{FOUR STEPS OF LATERAL PELVIC LYMPH NODE DISSECTION}

The LPND was performed consisting of 4 steps using surgical landmarks.

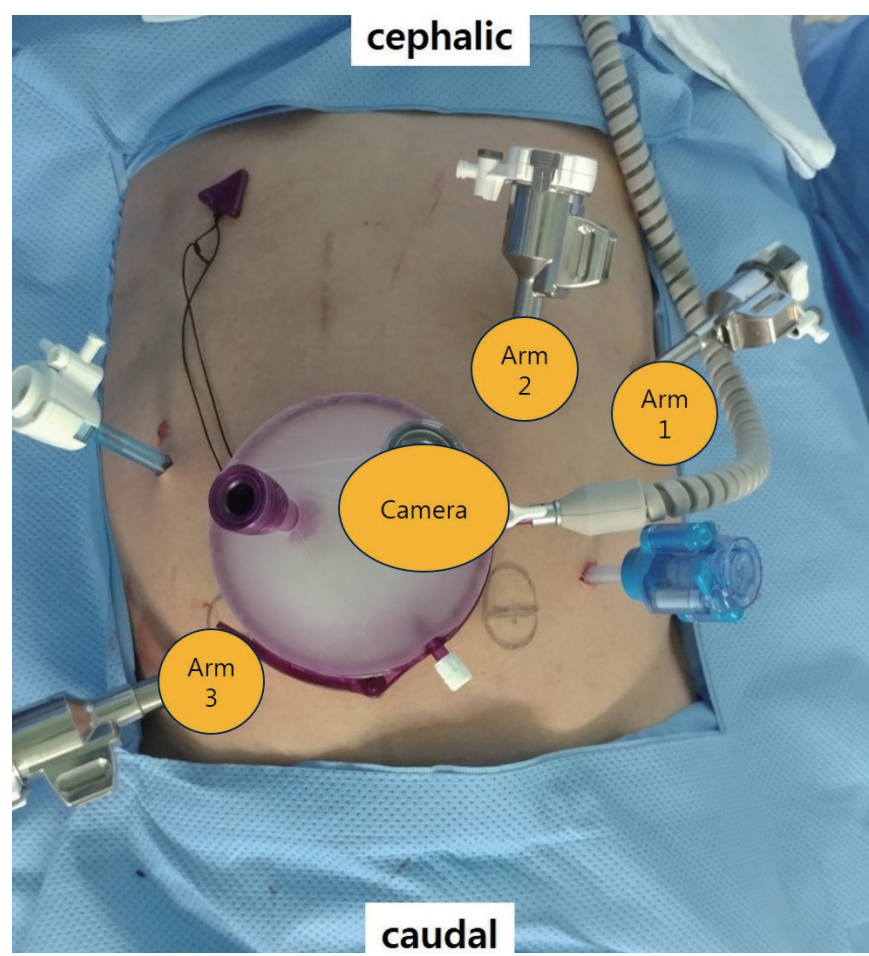

Fig. 3. Port sites in robotic lateral pelvic lymph node dissection. 


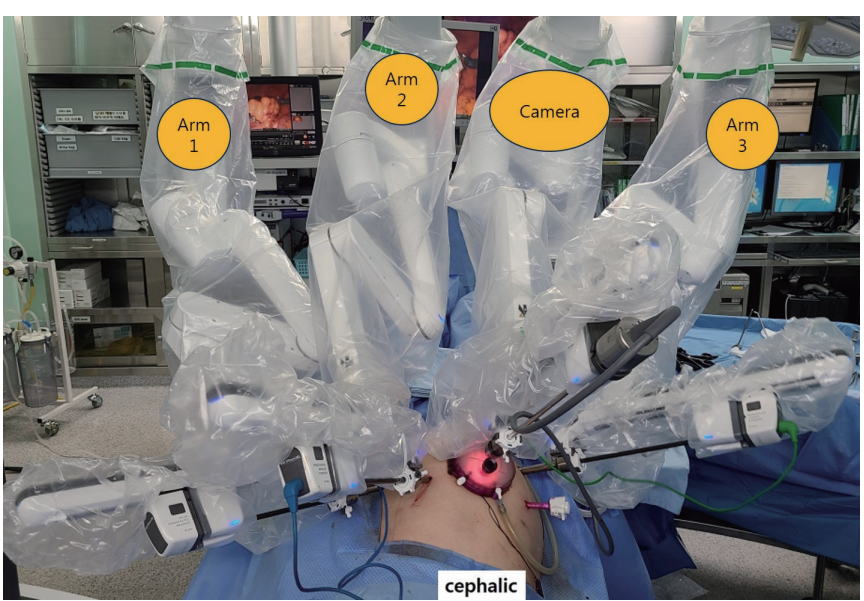

Fig. 4. Robot arm after docking in robotic lateral pelvic lymph node dissection.

The 1st step was the dissection of the uretero-hypogastric fascia, which envelopes the ureter, hypogastric nerve, and pelvic splanchnic nerve. Traction and countertraction was used to dissect the avascular plane.

The 2nd step was the dissection of the medial part of the external iliac vein, which is the lateral part of the obturator LNs group. After dissection of the external iliac vein, we identified the internal obturator muscle. We then identified the distal part of the obturator nerve, artery, and vein.

The 3rd step was the dissection of the vesico-hypogastric fascia, which surrounds the internal iliac vessels and inferior and superior vesical vessels. After dissecting around the common iliac artery bifurcation, we identified the proximal part of the obturator nerve. After dissecting the avascular plane of the lateral side of the umbilical artery, we reached the distal part of the obturator vessels and nerve, which were dissected at the second step. Afterward, the obturator LNs were removed.

The 4th step was the dissection of the internal iliac LNs group. We dissected the proximal part of internal iliac artery and continued along the internal pudendal artery. Finally, we identified the LNs around the Alcock's canal, which is the most distal part of the internal pudendal artery. Identification of this area is vital to a successful procedure as the Alcock's canal contains key LNs in communication with the lower rectum. The firefly mode was frequently used to identify the location of LNs. After LPND, we identified the skeletonized obturator nerve, artery, and vein, internal pudendal artery, and superior and inferior vesical arteries in the final surgical view. Finally, we checked the completeness of LPND using firefly mode. This video shows right-sided LPND. Left-sided LPND was likewise performed.

\section{SURGICAL OUTCOME}

The total operation time was 345 minutes with an estimated blood loss of $50 \mathrm{~mL}$. There were no intra- and postoperative complications, and the patient was discharged on postoperative day 4 . The final pathology was ypT3N1aM0. There were 7 lateral pelvic LNs harvested on the right side, and 5 lateral pelvic LNs on the left. No metastatic lateral pelvic LNs were identified.

\section{CONCLUSION}

This video showed a step-by-step technique using surgical landmarks in LPND by a robotic approach. We recommend this surgical technique as a standardized and convenient procedure for colorectal surgeons.

\section{SUPPLEMENTARY MATERIAL}

Supplementary video clip 1 can be found via https://doi.org/10. 3393/ac.2020.08.05.v001.

\section{REFERENCES}

1. Bosset JF, Collette L, Calais G, Mineur L, Maingon P, RadosevicJelic $\mathrm{L}$, et al. Chemotherapy with preoperative radiotherapy in rectal cancer. N Engl J Med 2006;355:1114-23.

2. Hashiguchi Y, Muro K, Saito Y, Ito Y, Ajioka Y, Hamaguchi T, et al. Japanese Society for Cancer of the Colon and Rectum (JSCCR) guidelines 2019 for the treatment of colorectal cancer. Int J Clin Oncol 2020;25:1-42.

3. Akiyoshi T, Ueno M, Matsueda K, Konishi T, Fujimoto Y, Nagayama S, et al. Selective lateral pelvic lymph node dissection in patients with advanced low rectal cancer treated with preoperative chemoradiotherapy based on pretreatment imaging. Ann Surg Oncol 2014;21:189-96.

4. Park JA, Choi GS, Park JS, Park SY. Initial clinical experience with robotic lateral pelvic lymph node dissection for advanced rectal cancer. J Korean Soc Coloproctol 2012;28:265-70.

5. Kang J, Yoon KJ, Min BS, Hur H, Baik SH, Kim NK, et al. The impact of robotic surgery for mid and low rectal cancer: a casematched analysis of a 3-arm comparison. Open, laparoscopic, and robotic surgery. Ann Surg 2013;257:95-101.

6. Currie AC, Brigic A, Thomas-Gibson S, Suzuki N, Moorghen M, Jenkins JT, et al. A pilot study to assess near infrared laparoscopy with indocyanine green (ICG) for intraoperative sentinel lymph node mapping in early colon cancer. Eur J Surg Oncol 2017;43: 2044-51.

7. Kawada K, Hasegawa S, Wada T, Takahashi R, Hisamori S, Hida $\mathrm{K}$, et al. Evaluation of intestinal perfusion by ICG fluorescence imaging in laparoscopic colorectal surgery with DST anastomosis. Surg Endosc 2017;31:1061-9. 